Recepción: 20 / 05/ 2018

Aceptación: 25 / 06 / 2018

Publicación: 01 / 08 / 2018

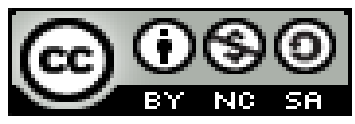

Educación Superior

Artículo Científico

\title{
La vinculación y su desafío con la comunidad desde la Educación Superior
}

The link and its challenge with the community from higher education

O vínculo e seu desafio com a comunidade do ensino superior

Myriam P. Loor-Vega
myriamloor@gmail.com

Fátima M. Figueroa Cañarte II

Fatima.m@gmail.com

María A. Quijije-Ortega III

mariaaortega@gmail.com

\author{
Edita M. Santos-Zambrano IV \\ editasantos@gmail.com \\ María A. Cedeño-Ugalde ${ }^{\mathrm{V}}$ \\ mariaacedeno@gmail.com \\ Derlin A. Delgado-Janumis VI \\ derlindelgado@gmail.com
}

Correspondencia: myriamloor@gmail.com

I. Magister en Gerencia de Salud para el Desarrollo Local; Licenciada en Ciencias de la Enfermería, Facultad de Enfermería, Universidad Laica Eloy Alfaro de Manabí, Manta, Ecuador.

II. Magister en Gerencia en Salud para el Desarrollo Local; Licenciada en Ciencias de la Enfermería, Facultad de Enfermería, Universidad Laica Eloy Alfaro de Manabí, Manta, Ecuador.

III. Magister en Gerencia de Salud para el Desarrollo Local; Licenciada en Ciencias de la Enfermería; Facultad de Enfermería, Universidad Laica Eloy Alfaro de Manabí, Manta, Ecuador.

Iv. Magister en Gerencia en Salud para el Desarrollo Local; Especialista en Gerencia y Planificación estratégica en Salud, Licenciada en Enfermería, Enfermera Profesional, Docente Universidad Técnica de Manabí, Jefa de Sala de Operaciones del Hospital General Portoviejo IESS, Portoviejo, Ecuador.

v. Magister en Epidemiologia; Doctora en Medicina y Cirugía, Carrera de Medicina, Facultad de Ciencias Médicas Universidad Laica Eloy Alfaro de Manabí, Manta, Ecuador.

vi. Magister en Salud Publica Mención Gerencia de los Servicios de Salud; Licenciada en Enfermería, Carrera de Enfermería, Facultad Ciencias de la Salud, Universidad Estatal del Sur de Manabí, Jipijapa, Ecuador. 


\section{Resumen}

Este articulo tiene por objeto estudiar un acercamiento sobre la responsabilidad social de la institución de educación superior, los desafíos y su vinculación con la sociedad, al servicio comunitario frente al desarrollo de la comunidad y la participación comunitaria, en todas sus características, contextos que tienen importancia en la adecuación de los programas de las carreras de las instituciones educativas hacia las necesidades de la realidad social y productiva en el ámbito en que se desenvuelve.

Definir los desafíos que la comunidad plantea permitiendo desarrollar la orientación y la gestión del currículo en la formación profesional en los últimos cursos de carrera propiciando el posicionamiento institucional que la universidad tiene con los sectores sociales, productores y estudiantes en formación profesional, que explore la necesidad de vinculación que la universidad tiene con el entorno globalizado.

Palabras claves: Educación superior, responsabilidad social, vinculación con la sociedad, participación comunitaria. 


\begin{abstract}
This article aims to study an approach on the social responsibility of the institution of higher education, the challenges and their relationship with society, community service in the face of community development and community participation, in all its characteristics, contexts that have importance in adapting the careers programs of educational institutions to the needs of the social and productive reality in the field in which it operates.

Define the challenges that the community poses by developing the orientation and management of the curriculum in professional training in the last years of career, promoting the institutional positioning that the university has with the social sectors, producers and students in professional training, that explore the need of connection that the university has with the globalized environment.
\end{abstract}

Key words: Higher education, social responsibility, links with society, community participation. 


\section{Introducción.}

La última década del siglo pasado fueron tiempos de las transformaciones por lo que la educación superior no fue la excepción, en este sentido, despertó el interés por la competencia dando prioridad a las respuestas de los requerimientos mediatos e inmediatos de las demandas del conglomerado social.

La Educación superior en la actualidad frente a los procesos de globalización, ha provocado un continuo y acelerado cambio, tales como la relación entre la Universidad-Estado-Sociedad, esto implica un gran desafío pues la universidad tiene una función social que interviene y transforma su entorno marcando su desarrollo, con un profundo compromiso ético con la sociedad, la modernización e introducción de nuevas tecnologías en el sistema productivo ayudará a construir una sociedad más justa.

El gran reto de la educación superior es que frente a la actual situación en la que se desenvuelven los sistemas educativo universitario, primordialmente las de carácter público, en un período de crisis económica, la gestión institucional es defectuosa y de deficiente eficacia, las técnicas de enseñanza-aprendizaje se ejecutan en situaciones precarias en términos de infraestructura; situación por lo que las instituciones de educación superior enfrentan una serie de desafíos de variada condición.

Situación que se contrapone al nuevo profesional en formación cuando el estudiante tiene que trabajar en una educación emprendedora es decir aprender a aprender y aprender a emprender, puesto que requiere de conocimientos y competencias, y es en este ámbito en el que aprenderá a generar oportunidades de emprendimiento. 
A fin de alcanzar los objetivos la universidad, la enseñanza se hace cada día más compleja y el aprender es una experiencia en esta caso se trata del "aprender haciendo", es una actividad desafiante para el alumno el mismo que debe responder a las necesidades educativas y sociales.

Por lo tanto podemos decir que para el caso de la educación superior, los profesores o tutores elaboran los sílabos, planifican, organizan, gestionan e implementan en el aula o en el área procesos de enseñanza-aprendizaje, acciones que ejecuta de acuerdo con su formación, experiencia, así como con los recursos con que cuenta en el contexto donde se desempeñan.

Vale reconocer que el proceso de enseñanza no es una labor sencilla, donde el tutor a más de los conocimientos teóricos debe poseer también conocimientos prácticos, tener además habilidades cognitivas y sociales, destrezas, actitudes, aptitudes y valores deseables, así como buena fracción de sentido común y perspicacia entre otras características, todo esto como componentes esenciales a fin de lograr su propia concreción, ubicándolo en situación más acorde con las exigencias que plantea la sociedad.

Las exigencias que las comunidades de la sociedad ejercen sobre las Instituciones de educación superior, las mismas que deben otorgar programas académicos, con formación humanística de sus futuros profesionales; así como, de que manera se diseña el desarrollo de sus procesos de enseñanza-aprendizaje y su vinculación al medio social y productivo; se deben implantar argumentos para considerar y buscar alternativas con el firme propósito de hacer efectivo los proyectos los mismos que deben ser tutorados $\mathrm{y}$, a través de los procesos de enseñanzaaprendizaje se llegue a reeducar a los beneficiarios para que también se vuelvan emprendedores, esto como un objeto de estudio en varios niveles de ampliación dada su alta complejidad; 
permitiéndoles estar al tanto en todas sus áreas y elementos de su desempeño que le permitan validar su eficacia y eficiencia, la pertinencia y responsabilidad social.

Esta orientación les permitirá encaminarse a la construcción de metas, mecanismos y procesos que promueven la pertinencia curricular de la universidad fortaleciendo la formación de los recursos humanos, estableciéndose una reconocida esencia estrechamente ligada a la dinámica social, correspondiendo con responsabilidad social al desarrollo comunitario y consecuentemente la participación comunitaria.

Ante ello es imprescindible reconocer, recapacitar y reenfocar el momento social en que se desenvuelve la universidad como nivel generador de mejora continua de la calidad de vida de la comunidad en la que se encuentran incorporadas instituciones responsables de la organización, ejecución y proyección la vida política y social del entorno, con el firme propósito de reafirmar la responsabilidad social.

\section{Desarrollo}

La educación superior y su responsabilidad social

UNESCO, (2009), La responsabilidad social de la educación superior

1. La educación superior, en tanto que bien público, es responsabilidad de todas las partes interesadas, en particular de los gobiernos.

2. Ante la complejidad de los desafíos mundiales, presentes y futuros, la educación superior tiene la responsabilidad social de hacer avanzar nuestra comprensión de problemas polifacéticos con dimensiones sociales, económicas, científicas y culturales, así como nuestra capacidad de hacerles frente. La educación superior 
debería asumir el liderazgo social en materia de creación de conocimientos de alcance mundial para abordar retos mundiales, entre los que figuran la seguridad alimentaria, el cambio climático, la gestión del agua, el diálogo intercultural, las energías renovables y la salud pública.

3. Los centros de educación superior, en el desempeño de sus funciones primordiales (investigación, enseñanza y servicio a la comunidad) en un contexto de autonomía institucional y libertad académica, deberían centrarse aún más en los aspectos interdisciplinarios y promover el pensamiento crítico y la ciudadanía activa, contribuyendo así al desarrollo sostenible, la paz y el bienestar, así como a hacer realidad los derechos humanos, entre ellos la igualdad entre los sexos.

4. La educación superior debe no sólo proporcionar competencias sólidas para el mundo de hoy y de mañana, sino contribuir además a la formación de ciudadanos dotados de principios éticos, comprometidos con la construcción de la paz, la defensa de los derechos humanos y los valores de la democracia.

5. Existe la necesidad de lograr más información, apertura y transparencia en lo tocante a las diversas misiones y actuaciones de cada establecimiento de enseñanza.

6. La autonomía es un requisito indispensable para que los establecimientos de enseñanza los puedan cumplir con su cometido gracias a la calidad, la pertinencia, la eficacia, la transparencia y la responsabilidad social.

Ayala G., Mauricio O., (2011), La responsabilidad social universitaria es saber administrar los impactos que la universidad tiene en sus cuatro áreas de funcionamiento (administración, formación, investigación y participación social). Así es necesario que sea responsable no solo de cara hacia su entorno a través de proyectos sociales que incluyen a estudiantes de varias 
especialidades, sino también en la manera en la que, por ejemplo, recicla sus desechos, rinde cuentas transparentes o cuida del personal administrativo y académico. No es sólo asegurar que sus estudiantes sean el día de mañana responsables, es también asegurarse de que ella misma lo es.

Zapata, W., (2014), la formación académica deberá ser integral, superando todo el lastre tradicional docentista, bajo una visión aglutinadora de las actividades académicas elaboradas a partir de la comprensión de que la esencia de la educación superior es la formación integral de las personas que acuden a ella. Si las instituciones de educación superior no forman de manera integral a sus alumnos, no cumplen su función ni su razón de ser.

En este contexto el compromiso de la universidad exige de su habilidad y efectividad para responder a las necesidades de transformación de la sociedad donde está inmersa, mediante el ejercicio de sus funciones básicas: docencia, investigación y extensión/proyección social. Estas funciones deben estar respaldadas por la búsqueda de la promoción de la justicia, la solidaridad y la equidad social, mediante la construcción de respuestas exitosas para atender los retos que implica promover el desarrollo humano integral (Guillén, 2012).

La comprensión integral e integradora de la formación académica posibilitará la construcción de un proyecto educativo que sea capaz de formar un sujeto crítico, con capacidad de globalización y acción en circunstancias cambiantes y críticas. De lo contrario, la educación superior continuará ofreciendo egresados parcialmente formados, inseguros y sin un horizonte claro; profesionales que creen que el trabajo en la sociedad consiste solo en la aplicación de unas cuantas técnicas y recetas memorizadas, en un mal entendido proceso de formación. Zapata, W., (2014).

En nuestros días se presenta una preocupación más o menos extendida de que las instituciones educativas, en lo general, no están cumpliendo a cabalidad con su responsabilidad de 
Myriam P. Loor-Vega; Fátima M. Figueroa Cañarte; María A. Quijije-Ortega; Edita M. Santos-Zambrano; María A. Cedeño-Ugalde; Derlin A. Delgado-Janumis

formar a las nuevas generaciones con las capacidades requeridas en una sociedad que se transforma profunda y aceleradamente (Chiappe \& Guido, 2009; Didriksson, s/f; Marcelo, 2001; Mella, 2003; Tedesco, 1996), citados por (Pérez, 2012).

Brünner, (2002) y Tedesco, (2000), coinciden en señalar que, a la par del veloz desarrollo de las tecnologías va tomando fuerza la idea de educación permanente y capacidad adaptativa al trabajo, lo que implica que será necesario educarse a lo largo de toda la vida para poder adaptarse a los requerimientos cambiantes del desempeño social y productivo.

La educación ha estado siempre estrechamente imbricada con la tecnología. Luego, así como se habla de las bases tecnológicas de un modo de producción -por ejemplo del modo de producción industrial- es posible, asimismo, hablar de las bases tecnológicas de la producción educacional. A fin de cuentas, la educación -como empresa social- es también una producción: la producción de un tipo humano determinado culturalmente o, si se quiere, la producción de unas competencias, unas disciplinas y unos conocimientos que necesitan ser comunicados e inculcados. Desde el punto de vista de la sociedad, se trata de la producción y reproducción del capital cultural distribuido entre individuos, familias, grupos y clases. Brunner, (2003).

Uno de los mayores desafíos de la Universidad actual, transita no por influir solamente en los acontecimientos sociales, sino en construir procesos sociales con identidad regional sostenibles que aseguren un verdadero empoderamiento desde la base. Para ello se debe entender cómo la Universidad redefine su responsabilidad para establecer un equilibrio entre las necesidades reales y el lograr incidir en la transformación de la comunidad, acabando con la organización separada del saber en especialidades y, al contrario, instituir el pensamiento holístico, complejo, 
transdisciplinario y práctico para formar una comunidad de aprendizaje asociativo con calidad y pertinencia. Cohen, (2007).

Carvajal, (2012), expresa que la RSU es: "la capacidad que tiene la Universidad de difundir y poner en práctica un conjunto de principios y valores generales y específicos”

Bueno y Casini, (2007), expresan que, el futuro de la «tercera misión» dependerá del grado de armonía e integración de objetivos y comportamientos del marco político-gubernamental, del marco social y empresarial y del marco académico-investigador de la universidad y de los Organismos Públicos de Investigación. Sincronía o diacronía que puede ser la clave para el éxito o el fracaso de la propuesta de la «tercera misión», como nueva perspectiva de la función de la Universidad en la actual sociedad del conocimiento.

Esta integración y cooperación responde a la metáfora, antes citada, del «modelo de triple hélice» o mejor, en lenguaje técnico-aeronáutico, de una hélice de «triple pala», en la que el giro coordinado de cada una de las palas que representan los agentes que protagonizan los actuales Sistemas de Ciencia, Tecnología y sociedad, es decir, universidades, gobiernos y empresas, podrá generar la energía necesaria para que el sistema de innovación despegue y se eleve lo más alto posible. Dadas las inercias, la complejidad y los recursos y capacidades que se ven involucrados en la definición y puesta en práctica con cierto éxito de la «tercera misión» de la Universidad para los próximos años. Bueno y Casini, (2007).

Los retos que estas manifestaciones plantean a la educación terciaria, están en marcha, en casi todas las regiones del mundo, procesos de transformación universitaria cada vez más profundos, que persiguen que la institución que llamamos "la Universidad" supere los nuevos retos y sobreviva, manteniendo incólume lo que ha sido hasta ahora su propia esencia. Así lo vislumbró la 
Myriam P. Loor-Vega; Fátima M. Figueroa Cañarte; María A. Quijije-Ortega; Edita M. Santos-Zambrano; María A. Cedeño-Ugalde; Derlin A. Delgado-Janumis

“Declaración Mundial sobre la Educación Superior para el Siglo XXI”, aprobada en París en 1998, cuando señaló que para responder a tales desafíos, las universidades debían emprender "la reforma más radical que jamás antes hayan enfrentado”. Tünnermann, (2011).

Según Martínez, (2007), la Universidad, debe contribuir a la solución de esos críticos problemas que aquejan a la sociedad y ellos deben percibirse a través de la identificación de necesidades sociales, económicas, políticas y asistenciales que son poco satisfechas, a través de las acciones que se han realizado y que requieren soluciones. Esa proyección social es la que cumple la Universidad con el medio y se apoya en sus actividades docencia - investigación de una manera interactiva, de una manera coordinada para que realmente pueda ser efectiva.

La responsabilidad social universitaria contribuye a clarificar y fortalecer la relación Universidad-Sociedad. Las cuatro funciones universitarias: gestión, docencia, investigación y extensión, cuando son ejercidas con una perspectiva ética, contribuyen a poner de manifiesto esa responsabilidad, de la que deben ser protagonistas todos los estamentos universitarios: directivos, administradores, docentes, investigadores, extensionistas $\mathrm{y}$, principalmente, los estudiantes. Consecuente con este criterio y de la necesidad de una integración creativa Universidad-Sociedad, existe la posibilidad de hacer de la responsabilidad social el eje de la acción universitaria. Tünnermann, (2011).

En este proceso la Universidad contribuye a reconocer dentro de estas necesidades lo que son las características, las culturas, las potencialidades necesarias y demandas que el medio esta cada día planteándole dispuesta a abrir múltiples y flexibles formas de interacción con esos sectores para darles también respuestas oportunas, deberá estar abierta y promoverá espacios de cooperación con otros centros, con otras universidades, con otros espacios, para que estas respuestas puedan ser un 
poco más congruentes y mucho más fortalecidas a través de las acciones que se ejercen. Martínez, (2007)

En estas consideraciones hay que dejar claro, que la promoción y divulgación de lo que hace la extensión es vital, hacer alianzas estratégicas con las empresas con otros organismos gubernamentales y no gubernamentales. Decirle a la sociedad, a la colectividad, esto es lo que hace la Universidad, esa es la contribución para el desarrollo regional y nacional, eso es extensión, buscar, conectarse con el entorno. Martínez, (2007)

Hernández y Saldarriaga, (2009), a partir de una investigación, analizan cómo las universidades, están preparando a los estudiantes para enfrentarse a la sociedad. Los mismos manifiestan que "La universidad se enfrenta entonces al problema de cómo llevar a la práctica lo que se predica y cómo evaluar el efecto de las prácticas tradicionales en la transformación social y el desarrollo de la región y el país que las acoge”.

Según Zapata, W., (2014), la formación de recursos humanos de excelencia académica y profunda sensibilidad social constituye la razón de ser de la educación superior, y el encargo social que debe asumir para desarrollar las cualidades intelectuales, humanas, culturales, artísticas, políticas y espirituales del estudiante, en conjunción con la naturaleza y la sociedad, articulando en forma orgánica y dinámica la docencia, la investigación, la vinculación con la sociedad, la difusión y la gestión académica, donde se entrelace razón y liberación.

\section{Desafíos de la Educación Superior y su vinculación con la sociedad}

Frente a los desafíos provenientes del conocimiento contemporáneo y de la sociedad global, es urgente estructurar las respuestas de las instituciones de educación superior. En primer lugar, y 
ante un mundo en constante proceso de cambio, la educación permanente aparece como la respuesta pedagógica estratégica que hace de la educación asunto de toda la vida y dota a los educandos de las herramientas intelectuales que les permitan "aprender a aprender" y adaptarse a los nuevos requerimientos del mundo social y laboral, y a la expansión y obsolescencia del conocimiento. Tünnermann, (2011).

Principales desafíos de la Educación Superior. Según Zapata, W., (2014)

Desafío 1.- Avanzar hacia la sociedad del conocimiento.

- $\quad$ Fortalecer al personal docente, administrativo y de autoridades

- Vincular la formación del talento humano de acuerdo a las demandas sociales, a más de los objetivos del Plan Nacional para el Buen Vivir.

- $\quad$ Plantear la sociedad del conocimiento mediante el fortalecimiento del conocimiento local en función de sus necesidades.

- Vincular el sector académico con los sectores de la producción.

Desafío 2.- Aportar al cambio de la matriz productiva que se centre en la producción de bienes y servicios y no a la exportación primaria.

- Incentivar la investigación científica en la universidad nacional mediante el cofinanciamiento de proyectos de investigación-acceso al Proyecto Prometeo, y del trabajo en conjunto con los Institutos Públicos de Investigación.

Desafío 3.- Evitar ahondar o reducir las brechas de desigualdad que aún persisten mediante una educación inclusiva. 
- Que la educación superior no excluya a nadie.

Desafío 4.- Asegurar una instrucción de calidad y adaptada a la realidad nacional.

- Que la educación superior esté orientada al cumplimiento de los objetivos del PNBV y especialmente centrada al hombre en la sociedad.

Desafío 5.- Contribuir a la generación de sentidos para que sus estudiantes sean reflexivos y se comprometan con la realidad del país.

- Que la educación superior forme profesionales con alto sentido de desarrollo personal proyectados hacia el servicio de la sociedad ecuatoriana.

Otros retos de la educación superior en el momento actual

- Generar el nuevo conocimiento

- Encontrar alternativas de solución o propuestas para reducir o eliminar problemas o dificultades identificas.

- $\quad$ Capacitación continua de la comunidad universitaria

- Ubicar la investigación científica en la malla curricular desde los primeros niveles.

- Intercambio continuo de docentes y estudiantes con otras universidades tanto a nivel nacional como internacional.

Para Sánchez, I., (2018), uno de los aspectos más destacados de la discusión fue el análisis de la vinculación de las universidades con sus egresados y con el territorio en las que están 
ubicadas. "Se hizo un gran énfasis en la responsabilidad de las instituciones en aportar al desarrollo de la sociedad, mediante la evaluación permanente de las necesidades del entorno y las vías de aportar a su crecimiento cultural y material, a través del trabajo conjunto con quienes han sido parte de sus aulas", explica el rector. Y, finalmente, enfatiza: "Las universidades debemos formar parte activa de la reflexión, aporte al acervo cultural, científico y artístico de nuestro país a través de nuestras comunidades y por la extensión de nuestros egresados, fruto tangible de nuestra presencia en el país".

El desafío de la universidad actual es refrendar su compromiso con la sociedad, preparando los recursos humanos que la misma necesita, para esto es necesario cerrar la brecha entre lo que se enseña en las universidades y lo que demanda el mercado laboral, estableciendo un constante monitoreo de la evolución de las empresas y ajustando constantemente los currícula de estudios como una respuesta a esas necesidades. Cuando las instituciones de educación superior hayan demostrado entonces que son capaces de generar el capital humano idóneo y capaz de enfrentar la realidad, las empresas tendrán menos desconfianza y habrá mayor probabilidad de establecer la vinculación vía proyectos empresariales. Saavedra, M. (2009).

El desafío de la universidad actual es refrendar su compromiso con la sociedad, preparando los recursos humanos que la misma necesita, para esto es necesario cerrar la brecha entre lo que se enseña en las universidades y lo que demanda el mercado laboral, estableciendo un constante monitoreo de la evolución de las empresas y ajustando constantemente los currícula de estudios como una respuesta a esas necesidades. Cuando las instituciones de educación superior hayan demostrado entonces que son capaces de generar el capital humano idóneo y capaz de enfrentar la realidad, las empresas tendrán menos desconfianza y habrá mayor probabilidad de establecer la vinculación vía proyectos empresariales. Saavedra, M., (2009). 
Según UNIVA, (2010), la globalización, los retos de las economías emergentes y de la educación superior; en la formación profesional competente, generación, aplicación y difusión del conocimiento científico y tecnológico y la investigación: que se desarrollan en la diversidad y dinamismo del ambiente en el cual interactúan. Obliga a que las universidades tengan características muy diferenciadas en sus modelos educativos e institucionales. Motivos por el cual los modelos de vinculación deben de estar vinculados al ser, quehacer y hacer de las economías, pero sobre todo de la academia, con adecuados canales de comunicación y coordinación, que permitan evaluar los resultados a partir de acciones que favorezcan a la superación de los retos que imperan en nuestro país como economía emergente.

UASD, (2012), indica que las universidades de Latinoamérica y El Caribe se enfrentan a desafíos para fortalecer su Pertinencia, Calidad, Eficiencia, Transparencia y Trascendencia en un ambiente de plena libertad y autonomía. Elevar la pertinencia implica para las instituciones de educación superior desarrollar investigaciones que generen conocimiento no solo de carácter utilitario, sino también cultivar la ciencia, la cultura, el humanismo sin que sus resultados se vean presionados por la necesidad utilitaria y mercantilista que se le atribuye hoy al conocimiento. Las universidades se enfrentan al criterio generalizado de que sólo es pertinente el conocimiento que tiene utilidad en el mercado.

También representa un gran desafío la pertinencia en cuanto a la formación profesionalizante, por el mismo utilitarismo mercantil del modelo napoleónico. Hoy se habla del papel de la educación superior de "formar los profesionales que demanda el mercado de trabajo" o formar el "capital humano" o los "recursos humanos", con lo que se reduce la formación profesional a formar "piezas" de una cadena de valor. Significa esto que el mercado no atribuye pertinencia a profesiones para las cuales no hay puestos de trabajo. El desafío es satisfacer esa demanda y al 
Myriam P. Loor-Vega; Fátima M. Figueroa Cañarte; María A. Quijije-Ortega; Edita M. Santos-Zambrano; María A. Cedeño-Ugalde; Derlin A. Delgado-Janumis

mismo tiempo ofrecer formación de profesiones que valoren nuestra diversidad humana y natural como nuestra principal riqueza. UASD, (2012).

Fernández, E., (2017), El reto, para el presente y para los años por venir, es resolver esos problemas desde una perspectiva integral, sostenible y sistémica, que facilite que los beneficios de este nivel educativo se extiendan a todos los sectores sociales y económicos. Ante este escenario, se plantea el desafío de si, para promover una renovación de la educación superior en nuestro país, es necesario un nuevo diseño institucional que atienda de forma integral a la complejidad y diversidad del sistema, así como a los nuevos retos que enfrenta y a la variada gama de actores involucrados.

Alcántar y Arcos, (2004), expresan que los cambios en la economía, los nuevos liderazgos militares y económicos, las propuestas de nuevos paradigmas que ha traído consigo la globalización, han motivado transformaciones sociales que han impactado a la educación superior. Ello, aunado a la escasez de recursos financieros de los gobiernos para apoyar a la educación; los cambios tecnológicos y de modelos educativos basados en el aprendizaje a lo largo de toda la vida; un mundo del trabajo más diversificado, con mayor competencia y nuevos requerimientos en destrezas, habilidades y conocimientos, ha obligado a las IES a buscar nuevas estrategias para responder adecuadamente a estos retos.

\section{La calidad académica a la vinculación al servicio comunitario}

La educación superior debe orientar la docencia a la formación de profesionales emprendedores que respondan proactivamente a las necesidades de los sectores productivos; que promocionen la investigación técnica y aplicada, y promuevan las vinculaciones con la comunidad, en un contexto guiado por valores éticos, la orientación a la calidad académica y al servicio comunitario. Zapata, W., (2014). 
Según Vásquez, (2012), la RSU es “un concepto novedoso, que lleva a interpretaciones diversas. Implica el compromiso de las Universidades en acciones concretas que respondan a necesidades realmente sentidas por la comunidad".

Fabara G., E., (2016), uno de los aspectos que debería tomarse en cuenta en una posible normativa para el futuro es la necesidad de incorporar más obligaciones y responsabilidades en el campo de la vinculación con la colectividad, puesto que los docentes universitarios del Ecuador deben tener una amplia conciencia social que permita a la institución educativa contribuir al mejoramiento de las condiciones de vida de los ciudadanos. A pesar de los esfuerzos realizados, en el país aún hace falta fortalecer una cultura nacional a favor de la educación superior e instaurar una profesión académica para el ejercicio de la docencia universitaria, con todas las implicaciones que tiene el desarrollo de una disciplina necesaria para la construcción del nuevo país.

Otra característica fundamental de la nueva formación profesional que esta modalidad educativa recoge es la vinculación escuela-empresa, con la finalidad de combinar los conocimientos adquiridos en el salón de clases con la práctica o el "aprender a hacer" en un centro de trabajo. La alternancia entre la escuela y el sector productivo permite a los estudiantes conocer el contexto real del trabajo e integrar conocimientos teóricos, tecnológicos y organizativos, lo que facilitará su posterior ingreso al mundo del trabajo. Silva, (2006).

El Artículo Noveno del Reglamento de Carrera y Escalafón del Profesor e Investigador del Sistema de Educación Superior establece las principales responsabilidades y funciones docentes, las cuales se concentran en los siguientes aspectos, CES, (2012).

10. "Diseño e impartición de cursos de educación continua o de capacitación y actualización;" Las instituciones de educación superior deben ser consideradas siempre como 
“organizaciones que aprenden”, en las que todos sus miembros deben fortalecer permanentemente su talento humano. Más aún se deben promover oportunidades de crecimiento y desarrollo para todos quienes forman las entidades educativas. La organización de eventos para la comunidad universitaria y para la colectividad cercana o afín a los propósitos de la institución es un trabajo permanente, que no debe dejar de realizarse siempre.

11. "Participación en actividades de proyectos sociales, artísticos, productivos y empresariales de vinculación con la sociedad articulados a la docencia e innovación educativa;" La universidad del siglo XXI no debe estar enclaustrada en sus cuatro paredes, debe hacer parte de los proyectos que se gestan al interior de la comunidad, debe promover la realización de proyectos de mejoramiento de las condiciones de vida, debe apoyar las iniciativas para fortalecer las organizaciones sociales y debe contribuir con sus conocimientos y experticia en la solución de los problemas ambientales, económicos, políticos y sociales.

López M., F., (2018), La vinculación obligatoria de la investigación al cambio de matriz productiva y a los planes de desarrollo se refuerza con el peso que, en el modelo de universidad que guía la reforma, se le asigna a la función de vinculación con la sociedad. Inspirada en la reforma de Córdova de 1918, que sustentó la creación de las denominadas extensiones de universitarias, la vinculación con la sociedad se ha convertido en una función de la universidad ecuatoriana tan importante como las funciones de docencia e investigación social.

Los impulsores de la reforma de Córdova habían visto a la universidad, más que como un espacio de formación e investigación, como un instrumento de cambio social. Esta idea ha llevado a un sobredimensionamiento de la capacidad de la universidad y, de manera específica, de la función de vinculación con la comunidad en la resolución de los problemas sociales e, incluso, tecnológicos 
del país. El impacto de la intervención de la universidad, a través de sus proyectos de vinculación con la comunidad, en la resolución de dichos problemas es mínimo. Y esto porque la responsabilidad y la capacidad para resolver dichos problemas no está en la universidad, sino en el Estado y la política pública. López M., F., (2018).

Se hace indispensable, de otro lado, reconociendo que las funciones esenciales de la universidad son la docencia y la investigación, redimensionar y reformular el alcance de vinculación con la sociedad, y situarla en el sitio que le corresponde. Es decir, no como una función autónoma y de igual importancia que la docencia y la investigación, sino como un mecanismo para reforzar tanto la una como la otra. López M., F., (2018).

Queda claro, no obstante, que las actividades de vinculación y sus resultados pueden promover el desarrollo de nuevas líneas de investigación y la adecuación de la oferta académica a las demandas sociales. Pueden, así mismo, fortalecer el papel de la universidad en el debate social y en la construcción de una agenda pública en relación con las distintas problemáticas que afectan al país. De hecho, recuperar la actoría social de la universidad pública es una tarea pendiente. Para recuperar el liderazgo en el debate público se requiere fortalecer la imagen institucional de la universidad. Algo que no podrá lograrse si antes no se confrontan las expectativas de cambio con la realidad e identidad histórica de la universidad ecuatoriana. López M., F., (2018).

\section{Desarrollo y participación comunitaria}

Casilla (2002), asume que la comunidad es una forma de interrelacionarse, compartiendo o no, intenciones, capacidades, acciones, espacios, circunstancias y proyectos de desarrollo colectivos, y que la participación comunitaria, es la acción humana de interacción e involucramiento en la consecución de un cambio o bien común. 
Ley de Servicio Comunitario del Estudiante de Educación Superior:

Art. 1. Normar la prestación del servicio comunitario del estudiante de educación superior, que a nivel de pre grado aspire al ejercicio de cualquier profesión.

Art. 4. A los efectos de esta Ley, se entiende por servicio comunitario, la actividad que deben desarrollar en las comunidades de estudiantes de educación superior que cursen estudios de formación profesional, aplicando los conocimientos científicos, técnicos, culturales, deportivos y humanísticos adquiridos durante su formación académica, en beneficio de la comunidad, para “cooperar con su participación al cumplimiento de los fines del bienestar social, de acuerdo con lo establecido en la Constitución de la República Bolivariana de Venezuela y en esta Ley.

Art. 5. La comunidad es el ámbito social de alcance nacional, estadal o municipal, donde se proyecta la actuación de las instituciones de educación superior para la prestación del servicio comunitario.

Art. 7. El servicio comunitario tiene como fines:

1. Fomentar en el estudiante la solidaridad y el compromiso con la comunidad como norma ética y ciudadana.

2. Hacer un acto de reciprocidad con la sociedad.

3. Enriquecer la actividad de la educación superior, a través del aprendizaje servicio, con la aplicación de los conocimientos adquiridos durante la formación académica, artística, cultural y deportiva. 
4. Integrar las instituciones de educación superior con la comunidad, para contribuir al desarrollo de la sociedad venezolana.

5. Formar, a través del aprendizaje servicio, el capital social en el país.

Art. 21. Los proyectos deberán ser elaborados respondiendo a las necesidades de las comunidades, ofreciendo soluciones de manera metodológica, tomando en consideración los planes del desarrollo municipal, estadal y nacional.

Hoy, los currículos suelen promover más el conocimiento teórico sobre los derechos humanos, la Constitución o las instituciones políticas, que el desarrollo de competencias vinculadas a la participación ciudadana activa. El desarrollo de proyectos de aprendizaje-servicio de calidad puede contribuir a brindar una Educación Cívica más centrada en el ejercicio de la ciudadanía y de una participación propositiva, conducente no sólo a bienintencionados cacerolazos, sino a soluciones efectivas para problemas demasiado tiempo postergados. (Tapia, 2002).

Acosta, (2013), expresa que las concepciones del Buen Vivir son ideas contemporáneas surgidas de las culturas indígenas y no indígenas que han establecido como fundamental las relaciones armónicas entre lo s seres humanos y la naturaleza. Particularmente el concepto andino propone en visualizar el desarrollo donde se construya nueva formas de vivir, en donde la cotidianidad, los aprendizajes son parte del cambio y de teorías nuevas donde se armonice la vida del ser humano con la naturaleza.

Según Peraza, (2005), el respeto a los derechos humanos es parte de la vinculación de la democracia participativa, ya que son elementos importantes para impulsar una democracia 
Myriam P. Loor-Vega; Fátima M. Figueroa Cañarte; María A. Quijije-Ortega; Edita M. Santos-Zambrano; María A. Cedeño-Ugalde; Derlin A. Delgado-Janumis

participativa, pero la ciudadanía con el aprendizaje continuo y efectiva participación ira fortaleciendo este modelo en la sociedad ecuatoriana.

Leon, (2014), indica que es la ciudadanía y las organizaciones sociales, las llamadas en aumentar la participación ciudadana desde una cultura de evaluación permanente y la búsqueda de la eficacia, estos son los desafíos de toda una sociedad.

\section{Conclusiones.}

En la actualidad la Educación superior frente a los procesos de globalización, le ha provocado un continuo y acelerado cambio, esto implica un gran desafío para la universidad, ya que con los nuevos escenarios y demandas sociales debe responder ante la comunidad interviniendo y transformando su entorno marcando su desarrollo.

la universidad tiene una función social que se fundamenta en la mejora continua en la calidad de sus procesos, alcanzando mantenerse en un comportamiento organizacional éticamente ejemplar constituyéndose en el estamento socialmente responsable ante la sociedad, la modernización e introducción de nuevas tecnologías en el sistema productivo ayudará a construir una sociedad más justa.

La globalización, ha permitido la transformación de la educación superior, precisamente cuando la educación esta basada en las competencias, esta importante realidad a motivado la transformación del conocimiento como motor de la economía y bienestar de la sociedad al mismo tiempo que la impulsa a la superación en la búsqueda de novedosas iniciativas.

La participación social de los involucrados permite la elaboración, organización, ejecución y evaluación de proyectos desde la institución de educación superior, permitiendo la vinculación para 
el desarrollo y por lo tanto puedan aprender juntos, es decir los participantes sean estos los educandos como los beneficiarios de determinado proyecto.

El Reglamento de Carrera y Escalafón del Profesor e Investigador del Sistema de Educación Superior del Ecuador, establece las principales responsabilidades y funciones docentes, en su Artículo noveno y literales 10 y 11, expresan que deben promover oportunidades de crecimiento y desarrollo para todos quienes forman las entidades educativas. La organización de eventos para la comunidad universitaria y para la colectividad cercana o afín a los propósitos de la institución es un trabajo permanente, que no debe dejar de realizarse siempre. Además, La universidad del siglo XXI no debe estar enclaustrada en sus cuatro paredes, debe hacer parte de los proyectos que se gestan al interior de la comunidad, debe promover la realización de proyectos de mejoramiento de las condiciones de vida, debe apoyar las iniciativas para fortalecer las organizaciones sociales y debe contribuir con sus conocimientos y experticia en la solución de los problemas ambientales, económicos, políticos y sociales.

Visto así, el desafío de la universidad es su compromiso con la sociedad, por lo tanto debe preparar los recursos humanos que la misma necesita, es de primordial importancia cerrar la brecha entre lo que demanda la comunidad y lo que se enseña en la educación superior, implantando procesos continuados de tutores implantando las directrices para una eficiente y eficaz ejecución de los proyectos, a fin de ir observando las falencias y ajustando a los currícula, esta sensibilización desde la universidad le permite promover nuevos procesos desde sus funciones y demostrando así acciones de responsabilidad social. 


\section{Biblioigrafia.}

Acosta, A., (2013), Buen Vivir, Sumak Kawsay, una oportunidad para imaginar otros mundos, Revista de Economía Mundial (33), 265

Alcántar, V. M. y Arcos, J. L., (2004), La vinculación como instrumento de imagen y posicionamiento de las instituciones de educación superior. Revista Electrónica de Investigación Educativa, 6(1). Consultado el día de mes de año en: http://redie.uabc.mx/vol6no1/contenido-enriquez.html

Ayala García, Mauricio Osvaldo, (2011), Responsabilidad social universitaria, <http://www.redicces.org.sv/jspui/bicscream/10972/91/1/Responsabilidad\%20social\%20 universitaria.pdf>

Brünner, José Joaquín, (2002), “Educación e Internet ¿La próxima revolución?”, Fondo de Cultura Económica, Chile.

Brunner, José Joaquín, (2003), Las nuevas tecnologías y el futuro de la educación, “La educación al encuentro de las nuevas tecnologías", UPE UNESCO / Septiembre Grupo Editor, Argentina.

Bueno Campos, Eduardo y Fernando Casani Fernández de Navarrete (2007), "La tercera misión de la universidad. Enfoques e indicadores básicos para su evaluación”, en Economía Industrial, núm. 366, pp. 43-59.

Carvajal, C., (2012), Responsabilidad Social Universitaria: Una introducción al concepto, de Ciudadanía y aprendizaje:

http://ciudadaniaaprendizaje.blogspot.com/2011/04/responsabilidad-social universitaria.html

Casilla, D., (2002), Tecnología Educativa para la Participación Comunitaria, Tesis Doctoral, Universidad del Zulia, Facultad de Humanidades y Educación.

CES, (2012), "Reglamento de Carrera y Escalafón del Profesor e Investigador del Sistema de Educación Superior”. Quito.

Cohen, Daniel, Desafíos de la Responsabilidad Social Universitaria. Razón y Palabra [en linea] 2007, 12 (Febrero-Marzo) : [Fecha de consulta: 26 de julio de 2018] Disponible en: $\leq$ http://www.redalyc.org/articulo.0a?id=199520735023>ISSN 1605-4806

Fabara Garzón, Eduardo, (2016). La formación y el ejercicio de la docencia universitaria en Ecuador. Desafíos. Alteridad, 11(2), pp. 171-181.

Fernández F., E., (2017), Una mirada a los desafíos de la educación superior en México, Innovación Educativa, ISSN: 1665-2673, vol. 17, número 74, 
Gaceta Oficial de la República Bolivariana de Venezuela. (2005), Ley de Servicio Comunitario para los Estudiantes de Educación Superior, No. 38.272 Año CXXXII-Mes XII, Caracas, 14 de Septiembre. Capítulo I, Art. 1; Capítulo II, Arts. 4, 5, 7; Capítulo IV, Art. 21.

Guillén, León; (2012), "La responsabilidad social universitaria: un estudio de caso aplicado a la Universidad Autónoma del Caribe en Barranquilla, Colombia", en Memorias VIII Congreso Internacional de Educación Superior Universidad 2012, La Habana, Cuba.

Hernández, R. D., y Saldarriaga, A., (2009), Gestión de la responsabilidad social universitaria, Caso: Escuela de Ingeniería de Antioquia-EIA, DYNA, Medellín.

Leon, J. G., (2014), DesafiosDeLaParticipacionCiudadana, www.dialnet. - 4796110.pdf

López Milán, Fernando, (2018), Los retos de la educación universitaria en el Ecuador, www.planv.com.ec /historias/sociedad/retos-la-educacion-universitaria-el-ecuador

Macías, L., y Pedroza, A., (2012), El reto de las universidades en sus modelos de vinculación universidad-empresa en economías emergentes; del modelo a la realidad, https://www.researchgate.net/publication/319989909_El_reto_de_las_universidades_en_sus _modelos_de_vinculacion_universidad-

empresa_en_economias_emergentes_del_modelo_a_la_realidad

Martínez de Carrasquero, Cynthia, (2007), "La responsabilidad social universitaria como estrategia para la vinculación con su entorno social", Brasil, Virtual Educa.

Peraza, A., (2005), Democracia participativa y derechos humanos, Aportes Andino N. 13, Gobernabilidad, democracia y derechos humanos.

Pérez Ruiz, Abel, (2012), "Desigualdad, mercado laboral y educación superior en América Latina", Universidad Autónoma Metropolitana Unidad Azcapotzalco, El Cotidiano, núm. 176, México.

Saavedra G., María L. (2009), Problemática y desafíos actuales de la vinculación universidad empresa: El caso mexicano, Actualidad Contable Faces, Año 12 No 19, Julio-Diciembre Mérida. Venezuela. Disponible en:〈http://www.redalvc.org/articulo.0a?id=25715409009> ISSN 1316-8533

Saavedra G., M. L. (2009), Problemática y desafíos actuales de la vinculación universidad empresa: El caso mexicano. Obtenido de Actualidad Contable FACES Año 12 № 19, Mérida, Venezuela.

Sánchez, Ignacio, (2018), Las universidades y el desafío de vinculación con sus egresados y la sociedad, Pontificia Universidad Católica de Chile, Santiago, Chile.

Silva Laya, Marisol, (2006), "La calidad educativa de las universidades tecnológicas: Su relevancia, su proceso de formación y sus resultados”, ANUIES, México. 
Myriam P. Loor-Vega; Fátima M. Figueroa Cañarte; María A. Quijije-Ortega; Edita M. Santos-Zambrano; María A. Cedeño-Ugalde; Derlin A. Delgado-Janumis

Tapia, M., (2002), Aprendizaje y servicio solidario: algunos conceptos básicos, Ministerio de Educación, Ciencia y Tecnología, Buenos Aires, Argentina. (Disponible en Línea) http://www.clayss.edu - cacióndigital.net

Tedesco, Juan Carlos, (2000), “Educar en la sociedad del conocimiento”, Fondo de Cultura Económica, Buenos Aires.

Tünnermann Bernheim, Carlos, (2011), “La educación superior frente a los desafíos contemporáneos”, Universidad Centroamericana, Nicaragua.

UASD, (2012), Desafíos de la educacion superior en Latinoamérica, https://www.uasd.edu.do/index.php/conferencia-rectoria/426-uasd/rectoria/conferencias/389popo, San Salvador, El Salvador. 6 de septiembre de 2012

UNESCO (2009), "La responsabilidad social de la educación superior", Conferencia Mundial sobre la Educación Superior-2009: La nueva dinámica de la educación superior y la investigación para el cambio social y el desarrollo, sede de la UNESCO, París.

<http://www.unesco.org/education/WCHE2009/comunicado_es.pdf>

Vásquez, G., (2012), Mitos y Objetivos de la Responsabilidad Social Universitaria, from IESALC UNESCO:

www.iesalc.unesco.org.ve/index.php?option=com_content\&view=article \&id=2596\%3

Amitos-y-objetivos-de-la-responsabilidad-social-universitaria\&catid=126\%3Anoticias-

pagina-nueva\&Itemid=712\&lang=es

Zapata Bustamante, Wilson, (2014), "Principales desafíos de la educación superior ecuatoriana", EcuadorUniversitario.Com 